Review

\title{
Large-Scale Simulations of Directed Self-Assembly with Simplified Model
}

\author{
Kenji Yoshimoto ${ }^{1 *}$ and Takashi Taniguchi ${ }^{2}$ \\ ${ }^{1}$ Center for the Promotion of Interdisciplinary Education and Research, Kyoto University, Japan \\ ${ }^{2}$ Department of Chemical Engineering, Kyoto University, Japan \\ *yoshimoto1@cheme.kyoto-u.ac.jp
}

\begin{abstract}
Directed self-assembly (DSA) is considered as a candidate for future patterning technology for semiconductor manufacturing. The DSA utilizes the phase separation of block copolymer and provides further resolution enhancement by the use of chemically and physically pre-patterned surface. In order for DSA to be a viable lithography solution, it is crucial to realize defect-free manufacturing process. In this study, we utilize the so-called Ohta-Kawasaki (OK) model to simulate the morphological defects of block copolymer formed on the chemically and physically pre-patterned surface. The OK model has advantages of the relatively low computational expense, scalability for large-scale simulations, and compatibility to the other simulation models such as self-consistent field theory (SCFT) through common physical properties of the materials. As test cases, we investigated the lamella defects of symmetric diblock copolymer formed on the chemically and physically pre-patterned surface. For the chemically pre-patterned surface, the two-dimensional (2D) dynamic simulations were performed including the thermal fluctuations, and the time evolution of the lamella defects was characterized as a function of the surface interactive parameter. In the three-dimensional (3D) dynamic simulations of the physically pre-patterned surface, effects of the trench width on the formation of the lamella defects was examined. Our preliminary results demonstrate that various types of the DSA lamella defects can be reasonably predicted by the OK model. It is expected that by calibrating the surface interactive parameters with experimental data, the OK model could be applied to various large-scale DSA simulations, e.g., hotspot analysis over a large area, and design/process optimizations with numerous parameters.
\end{abstract}

Keyword: DSA, Block Copolymer, Defect, Simulation, Model

\section{Introduction}

In the last several years, DSA has rapidly moved from university-level research to active development in many companies [1]. According to the International Technology Roadmap for Semiconductors 2012 Update [2], one of the major challenges for DSA is still defect-free processing. The morphological defects of diblock copolymer are formed dynamically during the annealing process. Since they are often turned into complicated 3D structures, DSA defects are difficult to be detected by only conventional methods using top-down SEM images [3].

Simulation could be a powerful tool to predict phase-separated morphologies of block copolymers on chemically and physically pre-patterned surface. Various simulation methods have been successfully applied to the DSA processes: self-consistent field theory (SCFT) [4,5], Monte Carlo (MC) methods [3], dissipative particle dynamics (DPD) [6,7], and coarse-grained molecular dynamics [8]. Due to the computational 
expense, however, these methods are generally not feasible for the large-scale DSA simulations, e.g., hotspot analysis over a large area, design and process optimizations including numerous parameters.

In this study, we investigated applicability of the so-called Ohta-Kawasaki (OK) model [9] to the DSA process. The OK model has been widely used for the morphological studies of polymeric and biological materials, taking an advantage of 1) the small number of the system variables, 2) readiness for the large-scale, static/dynamic simulations, and 3) compatibility to the other models through some common physical properties of the materials. As test cases, we utilized the OK model to simulate the lamella morphologies of symmetric diblock copolymer formed on the chemically and physically pre-patterned surface. Later in this paper, we also discussed the extensibility of the OK model.

\section{Model}

In the following, the lengths and energies are normalized by the radius of gyration, $R_{g}$, of the diblock copolymer and the thermal energy, $k_{B} T\left(k_{B}\right.$ : Boltzmann constant, $T$ : temperature), respectively.

\subsection{Ohta-Kawasaki Model}

In the OK model [9], the free energy, $F$, of diblock copolymer melt is described as

$$
F\{\eta(\boldsymbol{r}, t)\}=\int d r\left(f_{\text {mix }}+f_{\text {int }}+f_{\text {long }}\right),
$$

where

$$
\begin{aligned}
f_{\text {mix }} & =-\frac{1}{2 \xi^{2}} \eta(\boldsymbol{r}, t)^{2}+\frac{g}{4} \eta(\boldsymbol{r}, t)^{4}, \\
f_{\text {int }} & =\frac{1}{2}[\nabla \eta(\boldsymbol{r}, t)]^{2}, \\
f_{\text {long }} & =\frac{\alpha}{2} \int d \boldsymbol{r}^{\prime} G\left(\boldsymbol{r}, \boldsymbol{r}^{\prime}\right) \psi(\boldsymbol{r}, t) \psi\left(\boldsymbol{r}^{\prime}, t\right) .
\end{aligned}
$$

The order parameter, $\eta(\boldsymbol{r}, t)$, is the difference in local volume fractions between $A$ and $B$ segments, i.e., $\phi_{A}(\boldsymbol{r}, t)-\phi_{B}(\boldsymbol{r}, t)$, at position $\boldsymbol{r}$ and time $t$. The other order parameter, $\psi(\boldsymbol{r}, t)$, in Eq. (4) is defined by $\psi(\boldsymbol{r}, t)=$ $\eta(\boldsymbol{r}, t)-\bar{\eta}$, with $\bar{\eta}$ as the volume average of $\eta(\boldsymbol{r}, t)$. The mixing, interfacial and long-range energy terms of Eqs. (2-4) play a role of controlling the strength of micro-phase separation of diblock copolymer, the shape and size of the micro-phase separated domains, respectively. The coefficients, $1 / \xi^{2}$ in Eq. (2) and $\alpha$ in Eq. (4), are calculated from

$$
\begin{aligned}
\frac{1}{\xi^{2}} & =2 f(1-f)\left[2 \chi N-\frac{s(f)}{2 f^{2}(1-f)^{2}}\right], \\
\alpha & =3 / f(1-f),
\end{aligned}
$$

where $N$ is the total number of $A$ and $B$ segments per diblock copolymer chain (i.e., $N$ $\left.=N_{A}+N_{B}\right), f$ is the number fraction of $A$ segments in the diblock copolymer chain (i.e., $\left.f=N_{A} / N\right)$, and $\chi$ is the so-called Flory Huggins parameter. The function, $s(f)$, is empirically set at 1.33 at $f=0.1,1.00$ at $f=$ 0.3 , and 0.90 at $f=0.5$. The other coefficient, $g$, in Eq. (2) can be considered as a fitting parameter to the other theoretical models such SCFT (see Sec. 4.2). The Green function, $G\left(\boldsymbol{r}, \boldsymbol{r}^{\prime}\right)$, in Eq. (4) is satisfied with

$$
-\nabla^{2} G\left(\boldsymbol{r}, \boldsymbol{r}^{\prime}\right)=\delta\left(\boldsymbol{r}-\boldsymbol{r}^{\prime}\right) .
$$

\subsection{Surface Energy}

To describe the interactions between the diblock copolymer and the chemically pre-patterned surface, a surface energy, $F_{\text {surf }}$, is added to Eq. (1),

$$
F_{\text {surf }}\{\eta(\boldsymbol{r}, t)\}=\int d r \Lambda(\boldsymbol{r}) \eta(\boldsymbol{r}, t) .
$$

The surface interactive parameter, $\Lambda(\boldsymbol{r})$, is set to be a positive constant, $\Lambda_{s}$, on the attractive substrate to $B$ segments, and zero on the neutral surface.

\section{Simulation Methods}

\subsection{Time-Dependent Ginzburg Landau} (TDGL) Equation

A phase-separated morphology of the diblock copolymer at equilibrium can be obtained by minimizing the total free energy of a system, i.e., $F+F_{\text {surf }}$. In this study, the cell dynamics method [10,11] is employed, with which time evolution of the order parameter is numerically calculated from the TDGL equation;

$$
\frac{\partial \eta(\boldsymbol{r}, t)}{\partial t}=L \nabla^{2} \mu(\boldsymbol{r}, t)-\boldsymbol{\nabla} \cdot \boldsymbol{J}_{\boldsymbol{R}}(\boldsymbol{r}, t),
$$

where $L$ is a positive constant, and $\boldsymbol{J}_{\boldsymbol{R}}(\boldsymbol{r}, t)$ is the random flux caused by thermal fluctuations. The chemical potential, $\mu(\boldsymbol{r}, t)$, is obtained from the functional derivative of the free energy of the system with respect to 
the order parameter;

$$
\begin{aligned}
& \mu(\boldsymbol{r}, t)=\frac{\delta\left(F+F_{\text {surf } f}\right)}{\delta \eta(\boldsymbol{r}, t)} \\
& =\mu_{s}(\boldsymbol{r}, t)+\alpha \int d \boldsymbol{r}^{\prime} G\left(\boldsymbol{r}, \boldsymbol{r}^{\prime}\right) \psi\left(\boldsymbol{r}^{\prime}, t\right),(10)
\end{aligned}
$$

where

$$
\begin{aligned}
\mu_{s}(\boldsymbol{r}, t)=- & \frac{1}{\xi^{2}} \eta(\boldsymbol{r}, t)+g \eta(\boldsymbol{r}, t)^{3} \\
& -\nabla^{2} \eta(\boldsymbol{r}, t)+\Lambda(\boldsymbol{r}) .
\end{aligned}
$$

By substituting Eq. (10) into Eq. (9) and by making the first-order approximation, Eq. (10) is expressed as

$$
\begin{aligned}
\eta(\boldsymbol{r}, t+\Delta \tau) \approx & \eta(\boldsymbol{r}, t) \\
& +\Delta \tau\left[\nabla^{2} \mu_{s}(\boldsymbol{r}, t)-\alpha \eta(\boldsymbol{r}, t)\right] \\
& -\beta \sqrt{2 \Delta \tau / \Delta l^{3}} \boldsymbol{\nabla} \cdot \boldsymbol{J}_{\boldsymbol{R}}(\boldsymbol{r}, \boldsymbol{t}),
\end{aligned}
$$

where $\Delta \tau(=L \Delta t), \Delta l$ and $\beta$ are the time step, grid distance, and the magnitude of the thermal fluctuations, respectively. Note that in Eq. (12), the long-rang term is transformed into $\alpha \eta(\boldsymbol{r}, t)$ via Eq. (7). By this transformation, time-consuming calculations of the Green function in Eq. (10) can be eliminated.

\subsection{Simulation Flow}

Step 1: Setup of the initial conditions. The system was gridded with the distance, $\Delta l$, of $0.2\left[R_{g}\right]$. At each grid point, a random uniform number, $s(-0.02<s<0.02)$, was assigned as an initial value of the order parameter. The time step, $\Delta \tau$, was set at $4 \times 10^{-4}[\tau]$ for $2 \mathrm{D}$, $8 \times 10^{-6}[\tau]$ for $3 \mathrm{D}$. The physical properties of $A B$ diblock copolymer, $f$ and $\chi N$, were fixed at 0.5 and 14.0 , respectively. The coefficients, $1 / \xi^{2}$ and $\alpha$, were calculated from Eq. (5) and Eq. (6); $1 / \xi^{2}=10.4$ and $\alpha=12.0$.

Step 2: Calculations of $\mu_{s}(\boldsymbol{r}, t)$. Using the initial (or current) order parameter, the short-range chemical potential, $\mu_{s}(\boldsymbol{r}, t)$, was calculated from Eq. (11). The second derivative term, $\nabla^{2} \eta(\boldsymbol{r}, t)$, in Eq. (11) was estimated with the central difference method;

$$
\begin{aligned}
\nabla^{2} \eta(\boldsymbol{r}, t) \approx & {[\eta(x+\Delta l, y, z, t)+\eta(x-\Delta l, y, z, t)} \\
& +\eta(x, y+\Delta l, z, t)+\eta(x, y-\Delta l, z, t) \\
& +\eta(x, y, z+\Delta l, t)+\eta(x, y, z-\Delta l, t) \\
& -6 \eta(\boldsymbol{r}, t)] / \Delta l^{2} .
\end{aligned}
$$

Step 3: Calculations of $\nabla^{2} \mu_{s}(\boldsymbol{r}, t)$. The divergence of the thermodynamic flux of order parameter, $\nabla^{2} \mu_{s}(\boldsymbol{r}, t)$, was estimated by the same central difference method in Step. 2.

Step 4: Calculations of $\boldsymbol{\nabla} \cdot \boldsymbol{J}_{\boldsymbol{R}}(\boldsymbol{r}, t)$. At each time step, the $x y z$ components of the random thermal flux, $\boldsymbol{J}_{\boldsymbol{R}}(\boldsymbol{r}, t)$, were generated from a Gaussian distribution with the mean of 0 and the standard deviation of 1 . Then the divergence of the random thermal flux, $\boldsymbol{\nabla} \cdot \boldsymbol{J}_{\boldsymbol{R}}(\boldsymbol{r}, t)$, was also calculated from the central difference method;

$$
\begin{aligned}
& \boldsymbol{\nabla} \cdot \boldsymbol{J}_{\boldsymbol{R}}(\boldsymbol{r}, t) \approx \\
& {\left[J_{R x}(x+\Delta l, y, z, t)-J_{R x}(x-\Delta l, y, z, t)\right.} \\
& +J_{R y}(x, y+\Delta l, z, t)-J_{R y}(x, y-\Delta l, z, t) \\
& \left.+J_{R z}(x, y, z+\Delta l, t)-J_{R z}(x, y, z-\Delta l, t)\right] / 2 \Delta l
\end{aligned}
$$

Step 5: Update of $\eta(\boldsymbol{r}, t)$. The results from Steps 3-4 were input to Eq. (12) to calculate the new order parameter, $\eta(\boldsymbol{r}, t+\Delta \tau)$. Then the updated order parameter was fed back to Step 2. The iterative calculations were repeated over $1 \times 10^{6}$ time steps for the $2 \mathrm{D}$ simulations, and $2 \times 10^{6}$ time steps for the 3D simulations.

\subsection{Parallel Computing}

The simulation flow described in Sec. 3.2 can be implemented with parallel computing. Figure 1 illustrates a schematic flow diagram of the parallel computing. For simplicity, the thermal flux is not shown here. As a first step, the grids of the system are subdivided into $n$ blocks. Then, the time evolution calculations of $\eta(\boldsymbol{r}, t)$ at each of the subdivided blocks are separately performed on the one of the $n$ cores. At every time step, the order parameters and chemical potentials located at the edges of the subdivided blocks are transferred between the adjacent blocks. Such data transfer is required for the calculations of the second derivative terms as shown in Steps 2-4. If the block size is too small, the data transfer time becomes larger than the actual calculation time; the efficiency of the parallel computing is significantly degraded (see Sec. 4.1). In addition to the block size, the block shape is also an important factor to improve the efficiency of the parallel computing [12]. 


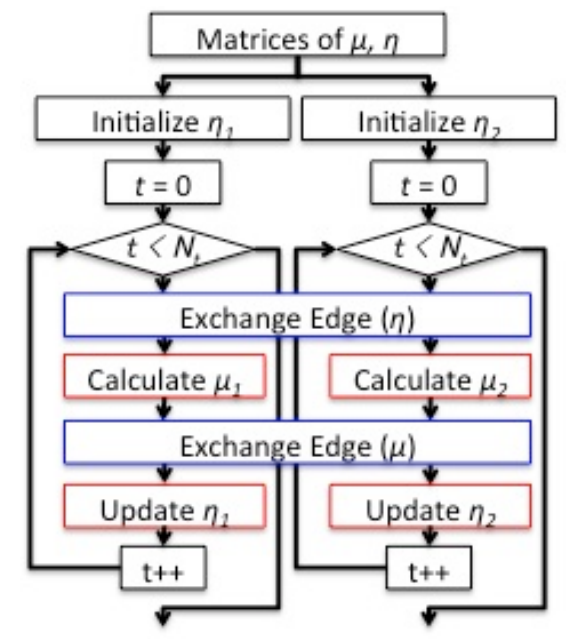

Figure 1. Schematic flow diagram of parallel computing.

\section{Results and Discussion}

\subsection{Scalability of Calculations}

Figure 2 shows an example of the speedup obtained by the MPI parallel computing for the $2 \mathrm{D}$ bulk lamella simulations over $10^{5}$ time steps. The 64 cores (i.e., 8 cores/processor $\mathrm{x} 8$ processors) were used here. The speedup factor was calculated by (computing time needed with single core) / (computing time with the 64 cores, including the data transfer time). It is clearly shown that the calculations were effectively parallelized, particularly for the larger systems. For the grid size of $2048 \times 2048$, the speedup was $\sim 55$, and the actual calculation time in each of the subdivided blocks was found to be much larger than the data transferring time. In general, the cell dynamic method used in this study is suitable for large-scale simulations with parallel computing [12].

\subsection{Comparison with SCFT}

The volume fraction of $A$ segments, $\phi_{A}$, in the bulk lamella structure is shown in Fig. 3. In the calculations of the OK model, the thermal fluctuations were not included. Also, the coefficient, $g$, in Eq. (2) was adjusted so that the maximum $\phi_{A}$ at $\chi N=12$ matched to that of SCFT. As shown in Fig. 3, the results of the OK model agree well with the density profiles of SCFT at $\chi N=11 \sim 14$, which is consistent with the previous study [13]. However, at $\chi N \gtrsim 14$, the peak of $\phi_{A}$ of the OK model became larger than unity, whereas the SCFT profile became shaper at the interface and flatter at the inside of the lamella domains (e.g., SCFT's profile at $\chi N=25$ in Fig. 3). Such a mismatch of the density profiles stems from the assumption of the OK model that the density deviation from the bulk average is considerably small. If one wants to fix this issue and extend the OK model to higher $\chi N$, a solution could be the use of the generalized Ohta-Kawasaki (gOK) model proposed by Uneyama and Doi [14] (See Sec. 3.6).

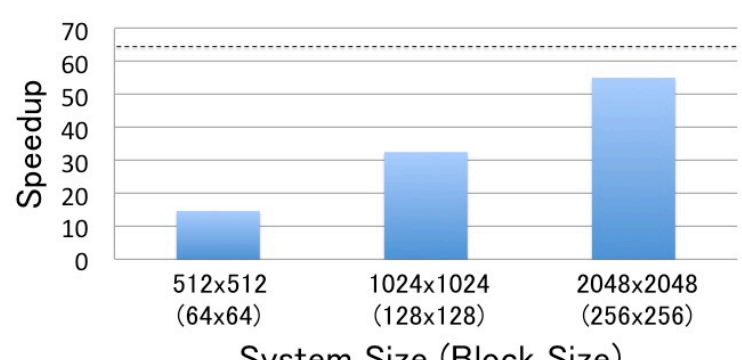

System Size (Block Size)

Figure 2. Speedup with parallel computing. The 64 cores were used for the $2 \mathrm{D}$ bulk lamella simulations. The dashed line denotes the ideal speedup, namely 64

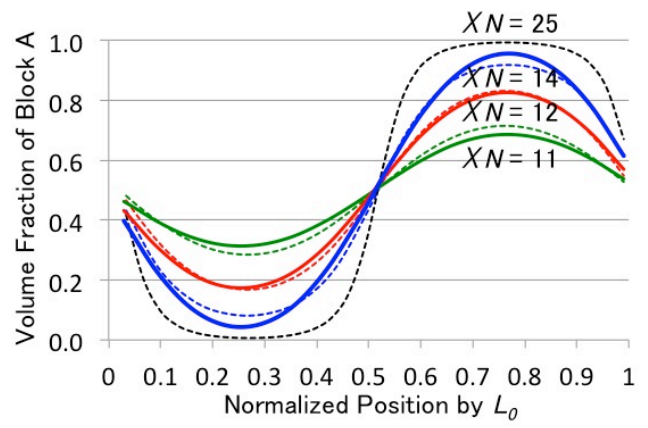

Figure 3. 1D density profile of the bulk lamellae, obtained from the SCFT (dashed) and the OK model (solid). The three different values of $\chi N$ were examined: 11 (green), 12 (red) and 14 (blue).

3.3. Effect of Thermal Fluctuations on Line Edge Roughness (LER) of Bulk Lamellae

The magnitude of the thermal fluctuations was adjusted by the coefficient, $\beta$, in Eq. (12). As shown in Fig. 4, the value of $\beta$ strongly affected on the LER of $2 \mathrm{D}$ bulk lamella structures. To be comparable to the actual LER of the PS-b-PMMA used in the DSA process, i.e., $\sim 2.5-3.5[\mathrm{~nm}]\left(\sim 0.5-0.7\left[R_{g}\right]\right)$ [15], the value of $\beta$ was chosen to be 0.1 in the following dynamic simulations. 


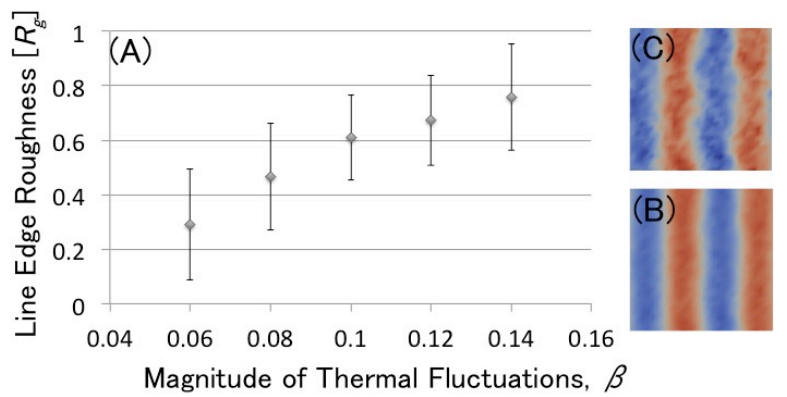

Figure 4. Line edge roughness vs. magnitude of the thermal fluctuations. On the right-hand side, the two images are the snapshots of the simulated lamella structures at (B) $\beta=0.06$ and (C) $\beta=0.14$.

3.4. Dynamics of Lamella Morphology on Chemically Pre-Patterned Surface

The chemically patterned surface was designed with the surface interactive parameter, $\Lambda(\boldsymbol{r})$, in Eq. (8). In this study, the values of $\Lambda(\boldsymbol{r})$ were set at a positive constant, $\Lambda_{s}$, on the chemical guide patterns, and zero at the outside of the guide patterns (Fig. 5). All the simulations for the chemically pre-patterned surface were performed in $2 \mathrm{D}$ with the grid size of $512 \times 512$ and under the periodic boundary conditions. Before introducing the chemical guide patterns, the native lamella pitch, $L_{0}$, was estimated by performing the Fourier transform of the fingerprint lamella morphology at $\Lambda_{s}=0$.

Figure 6 illustrates a dynamics of the simulated lamella morphologies on the 2D chemically pre-patterned surface. The width and pitch of the chemical guide patterns, $W$ and $P$, were set at $L_{0} / 2$ and $2 L_{0}$, respectively. The surface interactive parameter, $\Lambda_{s}$, was chosen to be 0.3 to represent the weakly attractive guide patterns to $B$ segments. At the beginning of the dynamic simulation, large clusters of the lamella defects were formed all over the place (Fig. 6A). Then the defects were gradually rearranged and turned into the straight lamellae (Fig. 6B). At the later stage, some localized lamella defects such as jogs and forks were sparsely observed (Fig. 6C). Those defects were eventually merged into the straight lamellae (Fig. 6D). Once the defect-free morphology was established, the straight lamellae were stabilized over a relatively long simulation time.

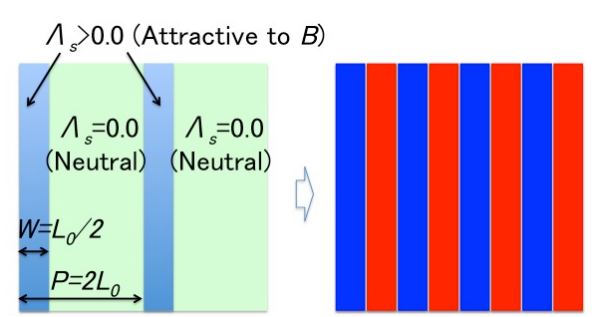

Figure 5. Schematic 2D designs of chemically pre-patterned surface.
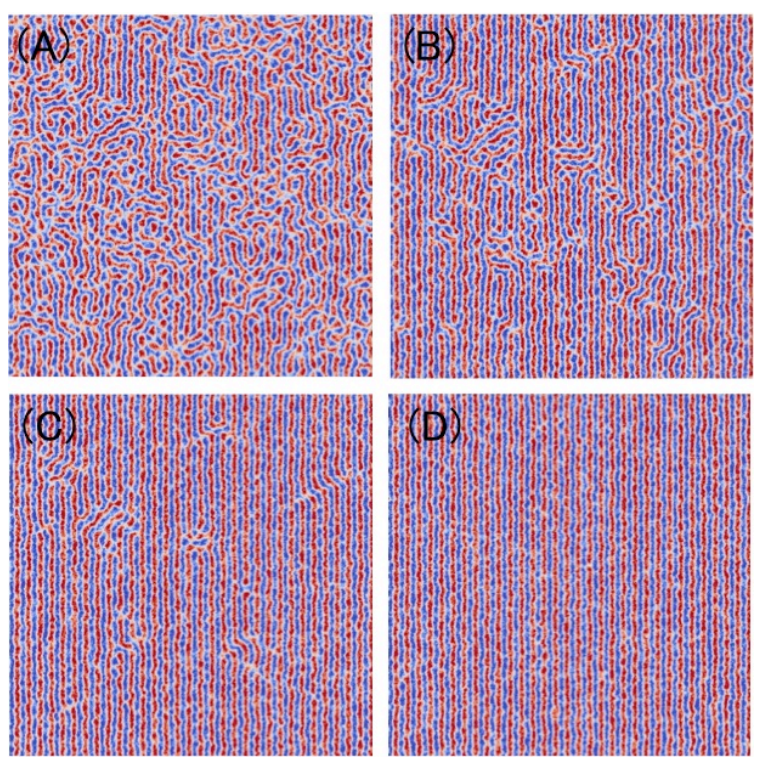

Figure 6. Dynamics of the DSA defects on the chemically pre-patterned surface: at (A) $t=4[\tau]$, (B) $t=8[\tau]$, (C) $t=12[\tau]$ and (D) $t=24[\tau]$, respectively. The $A$-rich and $B$-rich domains are shown by the red and blue colors, respectively.

By defining the defect fraction, $d$, as

$$
d=1-\frac{\# \text { of the defect-free lines }}{\text { of the ideal straigt lines }},
$$

the dynamic behavior of the lamella defects in Fig. 6 was characterized as a function of the simulation time, $\tau$. Fig. 7 illustrates three different curve of the defect fraction, obtained at $\Lambda_{s}=0,0.3,0.5$. It was found that the conversion from a random initial state to the defect-free state was facilitated by increasing $\Lambda_{s}$. Note that at $\Lambda_{s}=0$, the fingerprint morphology was held over a long course of dynamic simulations. Since similar analysis can be performed by taking the time-sequenced SEM images during the DSA annealing process [16], it is expected that the surface interactive parameter, $\Lambda_{s}$, and the simulation time scale, $\tau$, could be calibrated with experimental data. 


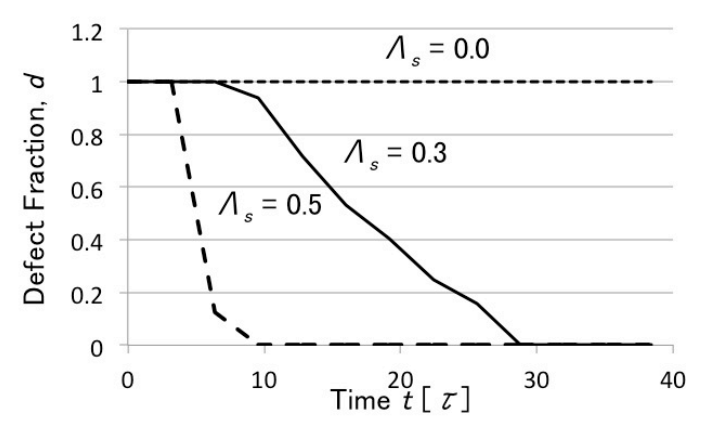

Figure 7. Change in defect fraction over annealing time: (dotted line) on the neutral surface, (solid line) on the chemically pre-patterned surface with the guide patterns weakly attractive to $B$ segments, and (dashed line) with the chemical guide patterns strongly attractive to $B$ segments.

\subsection{Lamella Morphology Generated on} Physically Pre-Patterned Surface

As a test case, the physically pre-patterned surface was designed with the trench depth of $L_{0}$ and the length of $12 L_{0}$ (Fig. 8A). The width of the trench, $W$, was changed from $6 L_{0}$ to $12 L_{0}$. The surface interactive parameter, $\Lambda(\boldsymbol{r})$, was set at 0.008 at the sidewalls, and zero at the top and bottom surfaces. In order to prevent from the volumetric flow going through the walls, the hard-wall boundary conditions, i.e., $\nabla \mu=0$ (only in the normal direction to the surface) [17], were applied on the sidewalls and on the top and bottom surfaces. The 3D simulations were performed without the thermal fluctuations over $2 \times 10^{6}$ time steps.

The resultant lamella morphologies in the trench are summarized in Fig. 8. The lamella defects were not observed at $W=6 L_{0}$ and $8 L_{0}$ (Fig. 8B and 8C), whereas the relatively small defects were formed at $W=10 L_{0}$ (Fig. 8D). At $W$ e $10 L_{0}$, large defects were formed in the middle of the trench (Fig. 8E), and stabilized over a long course of the simulations. Similar observations are reported in the literature [18], in which the PS-b-PMMA diblock copolymer was annealed in the trench made by the attractive sidewalls and the neutral bottom surface. The results showed that the large defects were formed near the center of the trench at $W$ e $\sim 8 L_{0}$.

\subsection{Extensibility of OK Model}

The OK model can also be applied to predict the cylindrical morphology of diblock copolymers in

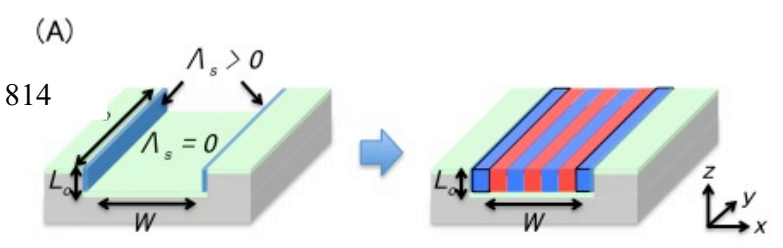

(B)

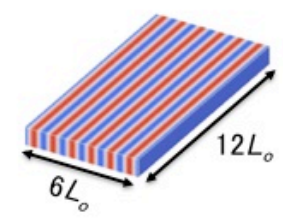

(D)

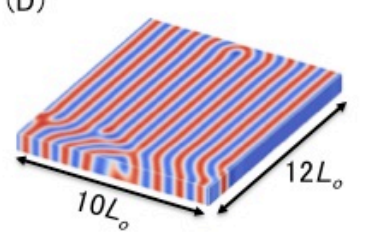

(C)

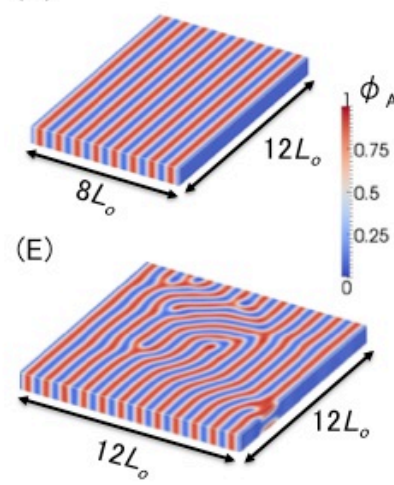

the confined geometry and on the chemically pre-patterned surface $[11,19]$.

However, as

Figure 8. Lamella morphologies on the physically pre-patterned surface: (A) schematic design of the trench, and (B-E) lamella morphologies obtained at different trench widths.

illustrated in Sec. 3.2, the OK model is not guaranteed to be comparable to the other models (e.g., SCFT and MC) at high $\chi N$. This is mainly due to the model assumption of the small density fluctuations around the bulk average. In case a discrepancy between the OK model and the other models becomes nontrivial for the high $\chi N$ system, one solution could be the use of the so-called generalized Ohta-Kawasaki (gOK) model [14]. The gOK model has a similar free energy formula to the original OK model; the free energy of the system is described as a function of the order parameter and is compromised of the mixing, interfacial, long-range, and one additional terms (see Ref. [14] for the details).

As an example, Fig. 9 illustrates the profiles of the volume fraction of $A$ segments, $\phi_{A}$, in the bulk lamella structure at $\chi N=25$ and $f=0.5$. The density profile of the gOK model was overlapped with that of SCFT. On the other hand, the density profile obtained from the $\mathrm{OK}$ model largely overshot the limits of $\phi_{A}$, i.e., 0 and 1 (thereby not shown in Fig. 9). 


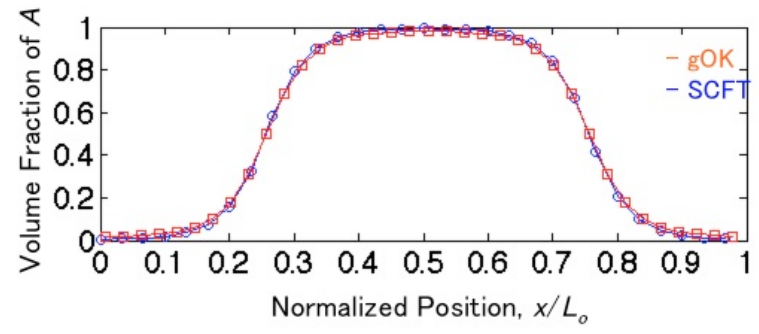

Figure 9. 1D density profiles of the bulk lamella structure at $\chi N=25$ and $f=0.5$, obtained from the generalized OK model (red) and the SCFT (blue).

In addition to high $\chi N$, the gOK model is also applicable to 1) arbitrary design of block copolymers (e.g., triblock, branched, star-shape), and 2) arbitrary blend of block copolymers, homopolymers, and solvents. It is important to note that all the coefficients in the gOK model can be predetermined from the physical properties of all compounds in the mixture, e.g., the degree of polymerization, block fraction, and $\chi$ parameters.

As a finial note, wall effects on the chain conformations of block copolymer were not included in this study. Such effects might not be negligible for the small confinements used in the DSA processes. We are currently investigating the confinement effects on the block copolymer morphology predicted by the OK and gOK models.

\section{Conclusion}

In this study, we simulated the DSA defects on the chemically pre-patterned surface, using a simplified model, so-called the Ohta-Kawasaki model. The model parameters were determined from the physical properties of the diblock copolymers, $\chi N$ and $f$. The simulated 1D density profiles of the lamella structure were comparable with those of SCFT at relatively low $\chi N$. For the chemically pre-patterned surface, the 2D dynamic simulations were performed, including the thermal fluctuations. The time evolution of the lamella defects was characterized as a function of the surface interactive parameter, $\Lambda_{s}$. In the 3D simulations of the physically pre-patterned surface, large lamella defects were formed in the middle of the wider trenches. It is expected that by calibrating the model parameters with experimental data, the OK model may be predictive of DSA defects over a large area. Our current works include the application of the generalized OK model to DSA process, which could extend the OK model to higher $\chi N$ and arbitrary blend system.

\section{Acknowledgements}

We would like to thank Yoshiro Hori for his assisting the parallel computing analysis. This work is supported by Japan Society of the Promotion of Science (JSPS) KAKENHI (Grant Number 25800237). K.Y. is also supported partly by Collaborative Research Program for Young Scientists of ACCMS and IIMC, Kyoto University.

\section{References}

1. D. J.C. Herr, J. Mater. Res., 26 (2011), 122.

2. ITRS website at http://www.itrs.net (2013).

3. F. A. Detcheverry, G. Liu, P. F. Nealey and J. J. de Pablo, Macromolecules, 43 (2010), 3446.

4. H. Takahashi, N. Laachi, K. T. Delaney, S. Hur, C. J. Weinheimer, D. Shykind and G. H. Fredrickson, Macromolecules, $\mathbf{4 5}$ (2012), 6253

5. T. Iwama, N. Laachi, K. T. Delaney, B. Kim, S.-M. Hur, R. Bristol, D. Shykind, C. J. Weinheimer and G. H. Fredrickson, $J$. Photopolym. Sci. Tec., 26 (1), 15.

6. T. Nakano, M. Matsukuma, K. Matsuzaki, M. Muramatsu, T. Tomita and T. Kitano, Proc. SPIE, 8680 (2013), 86801J.

7. H. Sato, H. Yonemitsu, Y. Seino, H. Kato, M. Kanno, K. Kobayashi, A. Kawanishi, K. Kodera and T. Azuma, Proc. SPIE, $\mathbf{8 6 8 0}$ (2013), 86801K.

8. R. A. Lawson, A. Peters, P. Ludovico and C. L. Henderson, Proc. SPIE, $\mathbf{8 6 8 0}$ (2013), $86801 \mathrm{Y}$.

9. T. Ohta and K. Kawasaki, Macromolecules, 19 (1986), 2621

10. Y. Oono, and S. Puri, Phy. Rev. Lett., 58 (1987), 836.

11. M. Pinna and A.V. Zvelindovsky, Eur. Phys. J. B, 85 (2012), 210.

12. X. Guo, M. Pinna and A. V. Zvelindovsky, Macromol. Theory Simul. 16 (2007), 779.

13. M. W. Matsen and F. S. Bates, Macromolecules, 29 (1996), 1091.

14. T. Uneyama and M. Doi, Macromolecules, 38 (2005), 196.

15. K. Patel, T. I. Wallow, H. J. Levinson and C. Spanos, Proc. SPIE, 7640 (2010), $76400 \mathrm{~T}$. 
16. A. M. Welander, K. O. Stuen, H. Kang, H. H. Solak, M. Muller, J. J. de Pablo and P. F. Nealey, Macromolecules, 41 (2008), 2759. 17. J. F. Marko, Phy. Rev. E, 48 (1993), 2861. 18. S. M. Park, M. P. Stoykovich, R. Ruiz, Y.
Zhang, C. T. Black and P. F. Nealey, $A d v$. Mater., 19 (2007), 607.

19. M. Pinna, X. Guo and A. V. Zvelindovsky, J. Chem. Phys., 131 (2009), 214902. 